\title{
Numerical Modelling of Transient Two-Phase Flows in a Fractured-Porous Medium
}

\author{
S. A. Khaliavin ${ }^{1,2}$, A. A. Afanasyev ${ }^{1,2}$ \\ ${ }^{1}$ Institute of Mechanics, Moscow State University \\ Moscow, 119192, Russia \\ ${ }^{2}$ Department of Mechanics and Mathematics, Moscow State University \\ Moscow 119991, Russia \\ semen14091996@gmail.com; afanasyev@imec.msu.ru
}

\begin{abstract}
We investigate transient two-phase flows in a fractured-porous medium. We assume existence of two different scales of the pore space, namely high-permeability fractures and low-permeability matrix blocks between these fractures. Transport in such medium can occur under local capillary disequilibrium between fractures and matrix blocks what is due to comparable time scales of capillary imbibition of the blocks and flows through the medium. In the paper, using an analytical approach, we determine distributions of the flow parameters in asymptotic cases corresponding to early and late stages of water injection into oil-saturated reservoirs. Using numerical modeling we investigate transient flows between the asymptotic cases.
\end{abstract}

Keywords: Porous medium, fractured reservoir, immiscible displacement, dual-porosity, effective single-porosity, transient flows

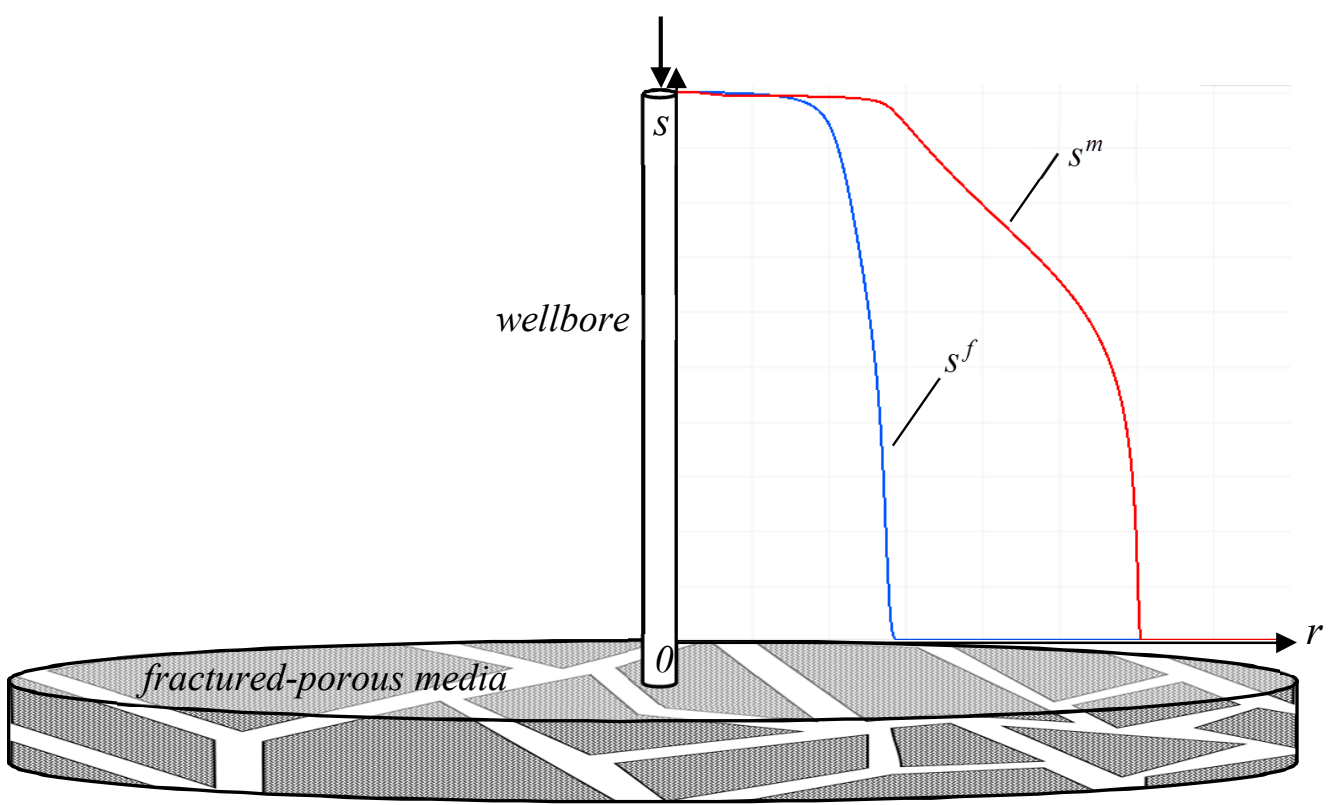

Schematic view of the axisymmetric problem of water injection into oil-saturated fractured reservoir. Typical distributions of water saturation in fractures, $s^{f}$, and, matrix blocks, $s^{m}$, are shown 


\title{
Численное моделирование переходных режимов двухфазной фильтрации в трещиновато-пористой среде
}

\author{
С.А. Халявин ${ }^{1,2}$, А.А. Афанасьев ${ }^{1,2}$ \\ ${ }^{1}$ НИИ механики МГУ имени М.В. Ломоносова, \\ Россия, Москва, 119192, Мичуринский пр., 1 \\ ${ }^{2}$ Механико-математический ф-т МГУ имени М.В. Ломоносова, \\ Россия, Москва, 119991, ГСП-1, Ленинские горы, д. 1 \\ semen14091996@gmail.com; afanasyev@imec.msu.ru
}

\begin{abstract}
Аннотация
Исследуются переходные режимы двухфазной фильтрации в трещиновато-пористой среде. Предполагается, что поровое пространство среды имеет различные масштабы высокопроницаемые трещины и расположенные между ними низкопроницаемые блоки. Фильтрация в подобных средах может происходить в отсутствии локального капиллярного равновесия между трещинами и блоками, что обуславливается сопоставимыми характерными временами капиллярной пропитки блоков и течения по среде. В работе аналитическими методами определены распределения параметров в асимптотических случаях, соответствующих начальным и поздним стадиям закачки воды в трещиноватопористую среду, насыщенную нефтью. Методом численного моделирования исследованы переходные режимы фильтрации между отмеченными асимптотическими распределениями.
\end{abstract}

Ключевые слова: трещиновато-пористая среда, фильтрация, двойная пористость, численное моделирование.

\section{1. Введение}

Рассмотрим одномерное двухфазное течение в трещиновато-пористой среде [1] (рис. 1). Введем параметр $n$, характеризующий геометрию течения: $n=1$ соответствует плоской постановке задачи, $n=2$ - осесимметричной, а $n=3$ - сферической симметрии течения. В частном случае $n=2$ схема течения изображена на рис. 1. Рассматривается горизонтальный пласт однородной трещиновато-пористой среды, который в начальный момент времени насыщен нефтью. Предполагается, что распределение трещин является однородным и изотропным, т.е. не зависит от направления. Через совершенную вертикальную скважину в пласт (т.е. в область $r>0$ ) с заданным расходом $Q$ нагнетается вода. Нагнетание воды приведет к развитию двухфазного течения в направлении от скважины в пласт, которое описывает вытеснение нефти водой. Практическая значимость исследования данного течения связана с тем, что схожие процессы происходят при заводнении нефтяных пластов.

Исследование течения будем проводить в рамках многоконтинуального подхода, в рамках которого трещиновато-пористая среда представляется в виде двух взаимопроникающих континуумов - $\Phi^{f}$ и $\Phi^{m}$. Первый континуум $\Phi^{f}$ соответствует трещинам, а второй $\Phi^{m}-$ низкопроницаемым блокам (рис. 2). В каждом континууме определены свои значения пористости, проницаемости и других параметров.

В начальный момент времени пласт насыщен нефтью, т.е. 


$$
t=0, r>0: s_{1}^{j}=0, s_{2}^{j}=1
$$

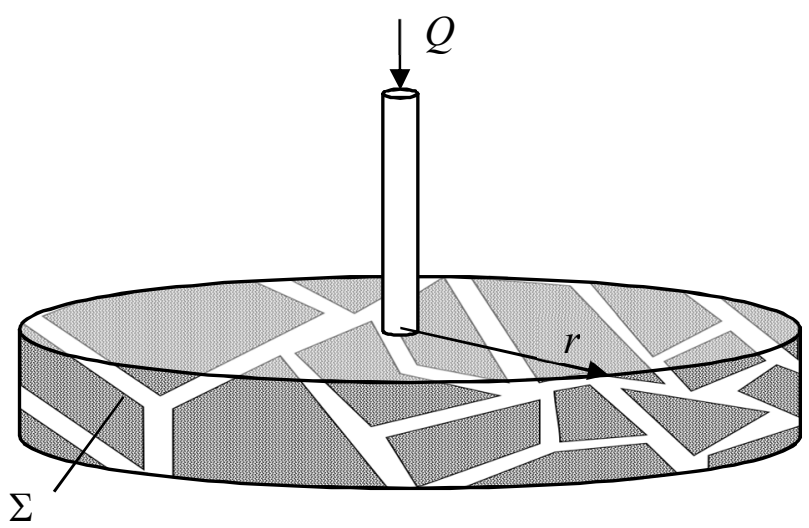

Рис. 1. Схема задачи при $n=2$

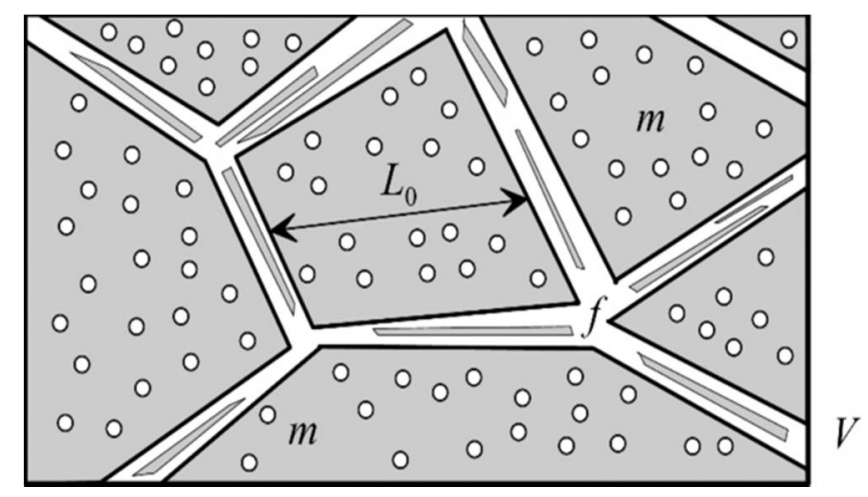

Рис. 2. Элементарный объем $V$ среды, содержащей трещины $f$ и низкопроницаемые блоки $m$. Характерный размер блоков $-L_{0}$

Здесь и далее $s$ - обозначает насыщенность фазы (т.е. ее объемную долю); нижний индекс $i=1,2$ обозначает параметры $i$-й фазы ( 1 - вода, $2-$ нефть), а верхний индекс $j=f, m-$ параметры в трещинах и блоках соответственно.

При $t=0$ в область $r>0$ через скважину начинается закачка воды с объёмным расходом $Q$ (см. рис. 1). Предполагается, что вода и нефть несжимаемые среды. Это означает, что объемный поток двухфазной смеси сохраняется при любом $r=$ const, t.e.

$$
Q=\int_{\Sigma} \Omega \mathrm{d} \sigma
$$

где $\Omega(r)$ - полная скорость фильтрации, т.е. сумма скоростей воды и нефти, а поверхность $\Sigma$ задается уравнением $r=$ const (см. рис. 1). Например, в случае $n=2 \Sigma$ есть поверхность цилиндра радиуса $r$. Следовательно, граничные условия имеют вид

$$
r \rightarrow 0: s_{1}^{f}=s_{1}^{m}=1, \boldsymbol{w}_{2}^{f}=\boldsymbol{w}_{2}^{m}=0, \boldsymbol{w}_{1}^{f}+\boldsymbol{w}_{1}^{m}=\frac{Q}{\Psi_{n} r^{n-1}},
$$

где $\boldsymbol{w}_{\boldsymbol{i}}$ - скорость фильтрации фазы $(i=1,2) ; \Psi_{n}$ - соответствующая симметрии течения константа: $\Psi_{1}=1, \Psi_{2}=2 \pi, \Psi_{3}=4 \pi$.

Постановка данной задачи схожа с постановкой классической задачи Баклея-Леверетта [1-3], а отличается от неё неравновесными процессами, обусловленными существованием двух масштабов порового пространства, соответствующих трещинам и низкопроницаемым блокам. Схожая постановка задачи, в частном случае $n=1$ рассматривалась в работе [4]. В настоящей работе исследуется общий случай произвольного значения параметра $n$.

\section{2. Основные уравнения}

\section{1. Замкнутая система уравнений}

Поровое пространство трещиновато-пористой среды охарактеризуем параметром $\gamma=V^{f} / V$ - относительным объёмом континуума $\Phi^{f}$, где $V=V^{f}+V^{m}-$ полный элементарный объём трещиновато-пористой среды, а $V^{j}, j=f, m$ - объём, относящийся к континууму $\Phi^{j}$ (см. рис. 2). Тогда относительный объём среды $\Phi^{m}$ равен $1-\gamma=V^{m} / V$. Для каждого континуума зададим пористость $\phi^{j}=V_{p o r}^{j} / V^{j}$ и абсолютную проницаемость $K^{j}$, где $V_{p o r}^{j}-$ объём порового пространства в соответствующем континууме. Тогда поровые пространства 
$\Phi^{f}$ и $\Phi^{m}$ занимают, соответственно, доли $\gamma \phi^{f}$ и $(1-\gamma) \phi^{m}$ элементарного объема $V$ соответственно.

Предполагая, что вода и нефть несжимаемые жидкости, уравнения фильтрации в трещиновато-пористой среде запишем в виде

$$
\begin{gathered}
\gamma \phi^{f} \frac{\partial s_{i}^{f}}{\partial t}+\nabla\left(\boldsymbol{w}_{i}^{f}\right)=q_{i}^{m f}, \quad i=1,2 \\
(1-\gamma) \phi^{m} \frac{\partial s_{i}^{m}}{\partial t}+\nabla\left(\boldsymbol{w}_{i}^{m}\right)=-q_{i}^{m f} \\
\boldsymbol{w}_{i}^{j}=-K^{j} \frac{K_{r_{i}}^{j}}{\mu_{i}} \nabla P_{i}^{j}, \quad j=f, m, \\
P_{2}^{j}-P_{1}^{j}=P_{c}^{j}\left(s_{1}^{j}\right), \\
q_{i}^{m f}=\sigma K^{m} \frac{K_{r_{i}}^{m f}}{\mu_{i}}\left(P_{i}^{m}-P_{i}^{f}\right),
\end{gathered}
$$

где $\phi$ - пористость; $\boldsymbol{w}$ - скорость фильтрации; $q^{m f}$ - объёмный поток из блоков в трещины; $K$ - проницаемость; $K_{r_{i}}$ - относительная фазовая проницаемость $i$-й фазы; $\mu=\mathrm{const}-$ вязкость; $P$ - давление; $P_{c}$ - капиллярное давление; $\sigma$ - параметр формы блоков [5]. Уравнения (3) и (4) - законы сохранения массы $i$-й фазы в трещинах и блоках соответственно, (5) - закон Дарси, соотношения (6) задают капиллярное давление, а (7) описывает массоперенос между континуумами $\Phi^{f}$ и $\Phi^{m}$. В каждом континууме насыщенности удовлетворяют соотношениям

$$
s_{1}^{j}+s_{2}^{j}=1, \quad j=f, m
$$

Обозначив $s^{f}=s_{1}^{f}$ и $s^{m}=s_{1}^{m}$, перепишем уравнения (3) и (4) в виде

$$
\begin{gathered}
(-1)^{i+1} \gamma \phi^{f} \frac{\partial s^{f}}{\partial t}+\nabla\left(\boldsymbol{w}_{i}^{f}\right)=q_{i}^{m f} \\
(-1)^{i+1}(1-\gamma) \phi^{m} \frac{\partial s^{m}}{\partial t}+\nabla\left(\boldsymbol{w}_{i}^{m}\right)=-q_{i}^{m f}
\end{gathered}
$$

Следуя [5], параметр формы блоков определяется следующим образом

$$
\sigma=\frac{4}{3 L_{0}^{2}},
$$

где $L_{0}$ - характерный размер низкопроницаемых блоков в изотропной среде (см. рис. 2).

Не ограничивая общности функции относительной фазовой проницаемости и капиллярного давления зададим в виде следующих функций от насыщенности фазы $(i=1)$ [4]:

$$
\begin{gathered}
K_{r_{1}}^{f}=s^{f}, \quad K_{r_{2}}^{f}=1-s^{f}, \quad P_{c}^{f}=0, \\
K_{r_{1}}^{m}=0.5\left(s^{m}\right)^{2.5}, \quad K_{r_{2}}^{m}=\left(1-s^{m}\right)^{1.5}, \quad P_{c}^{m}=P_{\max }\left(1-s^{m}\right), \\
P_{i}^{f} \geq P_{i}^{m}: \quad K_{r_{i}}^{m f}=K_{r_{i}}^{f}\left(s^{f}\right),
\end{gathered}
$$




$$
P_{i}^{f}<P_{i}^{m}: \quad K_{r_{i}}^{m f}=K_{r_{i}}^{m}\left(s^{m}\right),
$$

где $P_{\max }$ - константа, характеризующая максимальное значение капиллярного давления. Согласно равенствам (12), функции относительной фазовой проницаемости в объёме $\Phi^{f}$ равны насыщенностям соответствующих фаз, и капиллярное давление равно нулю $[5,6]$. В соответствии с (13), в соотношениях (7) для $q^{m f}$ относительные фазовые проницаемости $K_{n_{i}}^{m f}$ сносятся против потока. Если фаза $i$ течёт из $\Phi^{f}$ в $\Phi^{m}\left(q_{i}^{m f} \leq 0 ; P_{i}^{f} \geq P_{i}^{m}\right)$, то $K_{r_{i}}^{m f}=K_{r_{i}}^{f}\left(s^{f}\right)$, а если, наоборот, она течёт из $\Phi^{m}$ в $\Phi^{f}\left(q_{i}^{m f}>0 ; P_{i}^{f}<P_{i}^{m}\right)$, то $K_{r_{i}}^{m f}=K_{r_{i}}^{m}\left(s^{m}\right)$.

Подставляя соотношения (5), (7), (8), (12) и (13) в (6), (9) и (10), получим замкнутую систему пяти уравнений относительно неизвестных $s^{f}, s^{m}, P_{1}^{m}, P_{2}^{m}$ и $P^{f}=P_{1}^{f}=P_{2}^{f}$.

\section{2. Уравнения в безразмерном виде, параметры подобия}

Введем безразмерные параметры в виде

$$
r_{*}=\frac{r}{L}, \quad t_{*}=\frac{t}{T}, \quad w_{*}=\frac{w}{\Omega}, \quad P_{*}=\frac{P}{P_{0}},
$$

где звездочкой обозначены безразмерные переменные, а $L, T, \Omega, P_{0}$ - характерные масштабы длины, времени, скорости фильтрации и давления соответственно. Предполагается, что данные масштабы связаны следующими соотношениями:

$$
\begin{gathered}
Q=\Psi_{n} \frac{\bar{K} P_{0} L^{n-2}}{\bar{\mu}}, \quad L_{n}=\frac{Q T}{\bar{\phi} \pi}, \\
\bar{\mu}=\frac{2 \mu_{1} \mu_{2}}{\mu_{1}+\mu_{2}}, \bar{\phi}=\gamma \phi^{f}+(1-\gamma) \phi^{m}, \bar{K}=K^{f}+K^{m},
\end{gathered}
$$

где $\bar{\phi}, \bar{K}$ - эффективные (осредненные по масштабам $\Phi^{f}$ и $\Phi^{m}$ ) пористость и проницаемость соответственно.

Подставляя (5), (7), (14) и (15) в соотношения (3), (4) и (6) и "опуская" звёздочки у безразмерных величин, систему уравнений одномерной фильтрации в трещиновато-пористой среде представим в виде

$$
\begin{gathered}
(-1)^{i+1} \Gamma \frac{\partial s^{f}}{\partial t}+\frac{(1-\kappa) M_{i}}{r^{n-1}} \cdot \frac{\partial}{\partial r}\left(-K_{r_{i}}^{f} r^{n-1} \frac{\partial P^{f}}{\partial r}\right)=\frac{M_{i}}{\mathrm{~B}} K_{r_{i}}^{f m}\left(P_{i}^{m}-P^{f}\right), \\
(-1)^{i+1}(1-\Gamma) \frac{\partial s^{m}}{\partial t}+\frac{\kappa M_{i}}{r^{n-1}} \cdot \frac{\partial}{\partial r}\left(-K_{r_{i}}^{m} r^{n-1} \frac{\partial P_{i}^{m}}{\partial r}\right)=\frac{M_{i}}{\mathrm{~B}} K_{r_{i}}^{f m}\left(P_{i}^{m}-P^{f}\right), \\
P_{2}^{m}-P_{1}^{m}=C P_{c}^{m}, P^{f}=P_{1}^{f}=P_{2}^{f}, \\
\Gamma=\frac{\gamma \phi^{f}}{\bar{\phi}}, \kappa=\frac{K^{m}}{\bar{K}}, M_{i}=\frac{\bar{K} P_{0} L^{n-2}}{\mu_{i} Q}, B=\frac{1}{\kappa \sigma L^{2}}, C=\frac{P_{\max }}{P_{0}}, E=\frac{B}{C}
\end{gathered}
$$

параметры подобия.

Согласно (15) и (19), $M_{1}+M_{2}=2$. Следовательно независимым параметром можно выбрать только $M_{1}$, а параметр $M_{2}$ выражается через него: $M_{2}=2-M_{1}$. Параметр подобия $C$ характеризует влияние капиллярного давления на распределение насыщенности $s^{m}$ в континууме $\Phi^{m}$. Используя (15), его можно записать в виде 


$$
C=\frac{\Psi_{n} \bar{K} P_{0} L^{n-2}}{Q \bar{\mu}}
$$

Если $C \rightarrow 0$, то капиллярным давлением можно пренебречь.

Параметр подобия $B$ характеризует интенсивность массообмена между $\Phi^{f}$ и $\Phi^{m}$. Если $B \rightarrow 0$, то множители перед разностями давлений $P_{i}^{m}-P^{f}$ в правых частях уравнений (16) и (17) стремятся к бесконечности, а, следовательно, разности давлений стремятся к нулю (т.к. расход $Q$ конечный). Это означает, что при $B \rightarrow 0, C=0$ давления в средах $\Phi^{f}$ и $\Phi^{m}$ быстро выравниваются. Чем больше $B$, тем сильнее могут различаться давления $P_{i}^{f} \quad$ и $P_{i}^{m}$ в условиях отсутствия локального равновесия. Таким образом, $B$ характеризует неравновесность течения из-за различной динамики процессов в средах $\Phi^{f}$ и $\Phi^{m}$ при $C=0$.

Для оценки влияния капиллярного давления $(C \neq 0)$ на локальное равновесие, подставим соотношение (18) в уравнения (16), (17), исключив $P_{2}^{m}$ (или $P_{1}^{m}$ ). В результате получим, что в правой части $(16),(17)$ член перед $P_{c}^{m}$ обратно пропорционален отношению

$$
E=\frac{B}{C}=\frac{P_{0}}{\kappa \sigma L^{2} P_{\max }}=\frac{Q \bar{\mu}}{\Psi_{n} K^{m} P_{\max } L^{n}}
$$

Скорость установления капиллярного равновесия между $\Phi^{f}$ и $\Phi^{m}$ пропорциональна $1 / E$. При $E \rightarrow 0$ равновесие устанавливается мгновенно. Чем больше $E$, тем течение имеет более неравновесный характер из-за $P_{c}^{m} \neq 0$. Складывая попарно, при $i=1$ (или 2), уравнения (16) и (17), получим следующее следствие из системы (16)-(18):

$$
(-1)^{i+1} \frac{\partial \bar{s}}{\partial t}+\frac{M_{i}}{r^{n-1}} \cdot \frac{\partial}{\partial r}\left[r^{n-1}\left((1-\kappa) K_{r_{i}}^{f} \frac{\partial P^{f}}{\partial r}+\kappa K_{r_{i}}^{m} \frac{\partial P_{i}^{m}}{\partial r}\right)\right]=\frac{M_{i}}{B} K_{r_{i}}^{f m}\left(P_{i}^{m}-P^{f}\right),
$$

где

$$
\bar{s}=\Gamma s^{f}+(1-\Gamma) s^{m}
$$

эффективное значение насыщенности фазы $i=1$ в трещиновато-пористой среде. Уравнения (20) - законы сохранения массы $i$-й фазы, записанные для трещиновато-пористой среды в целом, с учетом обоих континуумов.

\section{4. Асимптотические случаи}

\section{1. Случай $X \rightarrow 0$ (начальная стадия закачки)}

Введём параметр $X=L / L_{0}$, характеризующий количество вовлечённых в течение блоков (т.е. блоков, находящихся за фронтом воды). В случае, когда $L_{0} \gg L$, параметр $X \rightarrow 0$. Это означает, что характерный размер блоков много больше характерного масштаба длины задачи. Исследуем фильтрацию в данном случае. Заметим, что параметры $\Gamma, \kappa, M_{i}$ не зависят от $X$, тогда как $B, C$ и $E$ зависят от $X$. Имеем

$$
\begin{gathered}
B=B_{0} X^{-2}, E=E_{0} X^{-n}, C=C_{0} X^{n-2}, \\
B_{0}=\frac{3}{4 \kappa}, \quad E_{0}=\frac{Q \bar{\mu}}{2 \pi K^{m} \sigma P_{\max } L_{0}^{2}}, \quad C_{0}=\frac{2 \pi \bar{K} P_{\max }}{Q \bar{\mu}},
\end{gathered}
$$

где константы $B_{0}, E_{0}, C_{0}$ не зависят от $X$. Убывание $X$ соответствует увеличению параметров $B$ и $E$, а течение происходит при отсутствии капиллярного равновесия. При $X \rightarrow 0$ параметры $B, E \rightarrow \infty$, а правыми частями уравнений (16), (17) можно пренебречь по сравнению с левыми частями, записав 


$$
\begin{aligned}
& (-1)^{i+1} \Gamma \frac{\partial s^{f}}{\partial t}+\frac{(1-\kappa) M_{i}}{r^{n-1}} \cdot \frac{\partial}{\partial r}\left(-K_{r_{i}}^{f} r^{n-1} \frac{\partial P^{f}}{\partial r}\right)=0 \\
& (-1)^{i+1}(1-\Gamma) \frac{\partial s^{m}}{\partial t}+\frac{\kappa M_{i}}{r^{n-1}} \cdot \frac{\partial}{\partial r}\left(-K_{r_{i}}^{m} r^{n-1} \frac{\partial P_{i}^{m}}{\partial r}\right)=0
\end{aligned}
$$

Таким образом, решение системы (16)-(18) сводится к двум независимым системам: уравнениям (22) относительно $P^{f}$ и $s^{f}$ и (18), (23) относительно $P_{i}^{m}$ и $s^{m}$. То есть задача распадается на две - задачу Баклея - Леверетта в трещинах (ее решение удовлетворяет (22)), и задачу Баклея - Леверетта в блоках (ее решение удовлетворяет (23) с соотношением на капиллярное давление (18)).

\section{2. Случай $X \rightarrow \infty$ (поздняя стадия закачки)}

В случае когда характерный масштаб длины $L$ много больше характерного размера блоков $L_{0}$ параметр $X \rightarrow \infty$. Это соответствует рассмотрению течения на больших пространственных масштабах. Возрастание $X$ соответствует установлению равновесного течения, так как при этом параметры $B$ и $E$ уменьшаются. В частном случае $n=2$ локальное равновесие из-за динамических процессов устанавливается наравне с равновесием, вызванным капиллярной разностью давлений: $B / E \sim 1$. В предельном случае $B \rightarrow 0(E \rightarrow 0)$, левыми частями уравнений (16), (17) можно пренебречь, записав

$$
K_{r_{i}}^{f m}\left(P_{i}^{m}-P^{f}\right)=0
$$

Уравнения (24) есть условия локального капиллярного равновесия между $\Phi^{f}$ и $\Phi^{m}$, так как если выполняется (24), то, согласно (7), перетоки $q_{i}^{m f}$ равны нулю.

Используя соотношения (13) и (24), определим при $C \neq 0$ равновесное распределение жидкостей $i=1,2$ между $\Phi^{f}$ и $\Phi^{m}$ в зависимости от эффективной насыщенности $\bar{s}$. Заметим, что при $\bar{s}=0$ насыщенности фазы $i=1$ равны нулю: $s^{f}=0, s^{m}=0$. Далее, так как $P_{c}^{m} \geq 0$ и $P_{c}^{f}=0$, то при возрастании $\bar{s}$ от нуля до единицы сначала фазой $i=1$ заполняется среда $\Phi^{m}$ (так как из-за капиллярного давления $P_{c}^{m} \geq 0$ фаза $i=1$ полностью впитывается в $\Phi^{m}$ из $\left.\Phi^{f}\right)$ и, когда $\Phi^{m}$ полностью заполнится (т. е. при $\left.s^{m}=1\right)$, начнет заполняться $\Phi^{f}$. Таким образом, учитывая (23), имеем

$$
\begin{gathered}
\bar{s}<1-\Gamma: s^{f}=0, \quad s^{m}=\frac{\bar{s}}{1-\Gamma}, \quad P_{1}^{m}<P_{2}^{m}=P^{f}, \\
\bar{s} \geq 1-\Gamma: s^{f}=\frac{\bar{s}-1+\Gamma}{\Gamma}, \quad s^{m}=1, \quad P_{1}^{m}=P_{2}^{m}=P^{f}
\end{gathered}
$$

В соответствии с (15), здесь учтено, что при $\bar{s}<1-\Gamma$ выполняются равенства $K_{\left(r_{1}\right)}^{f} m=0$ и $q_{1}^{m f}=0$, а при $\bar{s} \geq 1-\Gamma$ - равенства $K_{\left(r_{2}\right)}^{f} m=0$ и $q_{2}^{m f}=0$. Из уравнений (22) следует, что полный поток каждой фазы $i=1,2$ имеет вид

$$
M_{i} r \bar{K}_{r_{i}} \nabla \bar{P}_{i}=M_{i} r^{n-1}\left((1-\kappa) K_{r_{i}}^{f} \nabla P_{i}^{f}+\kappa K_{r_{i}}^{m} \nabla P_{i}^{m}\right)
$$

где $\bar{K}_{r_{i}}$ и $\bar{P}_{i}$ - эффективные относительные фазовые проницаемости и давления. Учитывая (25), положим, что при $\bar{s}<1-\Gamma$ выполняются равенства $\bar{P}_{1}=P_{1}^{m}, \bar{P}_{2}=P_{2}^{m}=P^{f}$, а при $\bar{s} \geq 1-\Gamma$ - равенства $\bar{P}_{i}=P_{i}^{m}=P_{i}^{f}$, тогда эффективные функции $\bar{K}_{r_{i}}$ и $\bar{P}_{i}$, удовлетворяющие (26), имеют вид 


$$
\begin{gathered}
\bar{K}_{r_{i}}(\bar{s})=(1-\kappa) K_{r_{i}}^{f}\left(s^{f}\right)+\kappa K_{r_{i}}^{m}\left(s^{m}\right), \\
C \bar{P}_{c}(\bar{s})=\bar{P}_{2}-\bar{P}_{1}=C P_{c}^{m}\left(s^{m}\right),
\end{gathered}
$$

где $s^{f}$ и $s^{m}$ выражаются через $\bar{s}$ из соотношений (27). Таким образом, подставляя (28) в (22), получим, что при $B, E \ll 1$ одномерная фильтрация в трещиновато-пористой среде описывается следующей системой уравнений относительно $\bar{s}, \bar{P}_{1}$ и $\bar{P}_{2}$ :

$$
\begin{gathered}
(-1)^{i+1} \bar{\phi} \frac{\partial \bar{s}}{\partial t}+\frac{M_{i}}{r^{n-1}} \cdot \frac{\partial}{\partial r}\left(\bar{K}_{r_{i}}(\bar{s}) r^{n-1} \frac{\partial \bar{P}_{i}}{\partial r}\right)=0, \\
\bar{P}_{2}-\bar{P}_{1}=C \bar{P}_{c}(\bar{s}),
\end{gathered}
$$

где фазовые проницаемости и капиллярное давление заданы в виде (27). Из решения уравнений (28) распределения параметров в средах $\Phi^{f}$ и $\Phi^{m}$ восстанавливаются по соотношениям (25).

Равновесная асимптотическая модель (28) проще полной модели (16)-(18), так как она позволяет свести исследование фильтрации в трещиновато-пористой среде к решению уравнений для эффективной одинарной среды, которое подробно рассмотрено в литературе $[2,3]$. Если в дополнение к условиям $B, E \ll 1$ выполняется неравенство $C \ll 1$, то в уравнениях (30) можно пренебречь капиллярным давлением, положив $P=P_{1}=P_{2}$. В этом случае исследование сводится к решению задачи Баклея-Леверетта, в которой насыщенность имеет автомодельное распределение от переменной

$$
\xi=r \sqrt[n]{\frac{\bar{\phi} \pi}{Q T}},
$$

т.е. $\bar{s}(r, t)=\bar{s}(\xi)$ [2, 3]. В случае общего положения из-за действия капиллярных сил $(C \nrightarrow 0)$ и неравновесных процессов $(E \nrightarrow 0)$ система уравнений (16)-(18) не имеет автомодельных решений.

\section{5. Течение при конечных $\boldsymbol{X}$ (переходные режимы)}

Ранее в разделах 4.1 и 4.2 был описан характер течения в двух предельных случаях: $X \rightarrow 0$ и $X \rightarrow \infty$. Важным также является рассмотрение того, каков характер течения при переходе от одного асимптотического случая к другому, т.е. исследование так называемых переходных режимов - течения двухфазной жидкости в трещиновато пористой среде при конечных $X$. Ранее в статье было установлено, что из всех безразмерных параметров от $X$ зависят лишь три $-B, E$ и $C$. То есть, в зависимости от значений данных параметров будут возникать различные переходные режимы. Далее подробно исследуется случай течения, когда $E_{0} \gg C_{0}$, т.е. когда существенен неравновесный массообмен между $\Phi^{f}$ и $\Phi^{m}$. Отметим, что в работе [4] при $n=1$ рассматривался случай $C_{0} \gg E_{0}$. Алгоритм проведения расчетов для переходных режимов при конечных значениях $X$ состоит из следующих шагов:

1. Задаются параметры $\gamma, \phi^{f}, \phi^{m}, K^{f}, K^{m}, \sigma, \mu_{1}, \mu_{2}, Q, P_{\max }$ и в соответствии с ними находятся значения параметров подобия (табл. 1).

2. С помощью первой формулы (15), которая связывает расход, время и размер расчетной области, определяется характерный масштаб течения $L$. Расчетное время $-T=400$ суток.

$$
L=\sqrt{\frac{Q T}{\bar{\phi} \pi}}
$$

3. Полученные параметры загружаются в гидродинамический симулятор MUFITS [7], где производится расчет нестационарного течения в указанной области длительностью $T$. 
В результате определяются распределения насыщенностей $s^{f}(r, t)$ и $s^{m}(r, t)$ и эффективной насыщенности $\bar{s}(r, t)$ от координаты $r$ в различные моменты времени $t$ (в том числе при $t=T$ ).

4. Используя соотношение (29) распределения насыщенностей строятся в зависимости от переменной $\xi$. Так как задача не автомодельная, то эти распределения различные при различных $t$ (и $X$ ).

\section{Таблиия 1}

\section{Значения размерных и безразмерных параметров в проведенных расчетах}

\begin{tabular}{|c|c|c|c|c|c|}
\hline$\gamma$ & 0.12 & $\sigma$ & 74667 & $\Gamma$ & 0.214 \\
\hline$\phi^{f}$ & 0.5 & $\mu_{1}$, Па.с & 0.001 & $M_{1}$ & 0.215 \\
\hline$\phi^{m}$ & 0.25 & $\mu_{2}$, Па.с & 0.01 & $\kappa$ & 0.7 \\
\hline$K^{f}, \mathrm{mD}$ & 300 & $Q, \mathrm{M}^{2} /$ сут. & 0.05 & $C_{0}$ & 0.09 \\
\hline$K^{m}, \mathrm{mD}$ & 700 & $P_{\max }$, бар & 0.01 & $E_{0}$ & 11.4 \\
\hline
\end{tabular}

В общем случае, возможны три режима с соответствующими значениями параметров подобия: $E_{0} \gg C_{0}$ (преобладание неравновесности в течении), $E_{0} \sim C_{0}$ (влияние неравновесности и капиллярного давления на течение одинаковы) и $E_{0} \ll C_{0}$ (преобладает влияние капиллярных сил на течение). Для удобства и наглядности возьмем логарифм по основанию 10 от $C$ и $E$ и построим графики полученных прямых в зависимости от $\log X$ (рис. 3 ). При $n=1$ графики $\log C$ и $\log E$ от $\log X$ параллельны (рис. 3, $a$ ). Следовательно, если $C_{0}>E_{0}$, то при любом $X$ влияние капиллярных сил преобладает над массообменом между средами (случай рассмотрен в [4]). Если же $C_{0}<E_{0}$, то наоборот неравновесный обмен при любом $X$ существеннее. При $n=2$ и $n=3$ (рис. 3, б и в) графики прямых $\log C$ и $\log E$ пересекаются при некотором $X_{*}$. Причем при $X<X_{*}$ преобладают неравновесные эффекты, а при $X>X *$ преобладает влияние капиллярных сил на распределение параметров в пространстве. Далее подробнее рассмотрен случай $n=2$ и $E_{0} \gg C_{0}$, так как он позволяет исследовать при малых $X$ - неравновесность течения, а при больших $X$ - преобладание влияния на течения капиллярных сил.

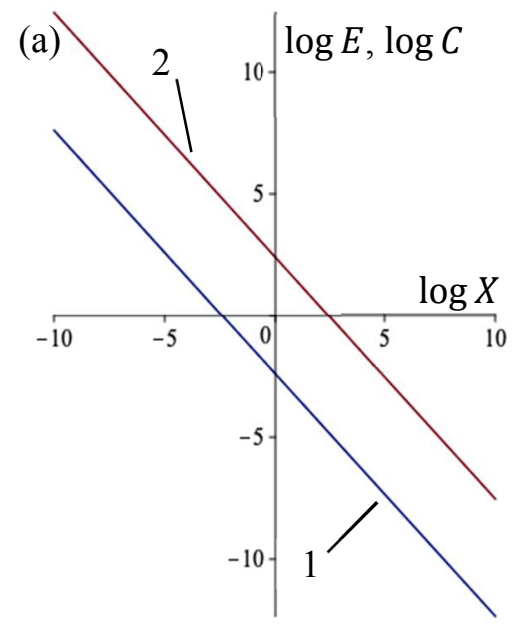

$a$

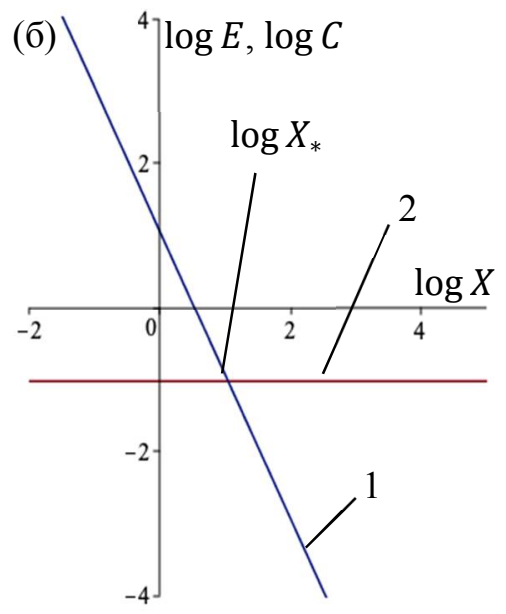

6

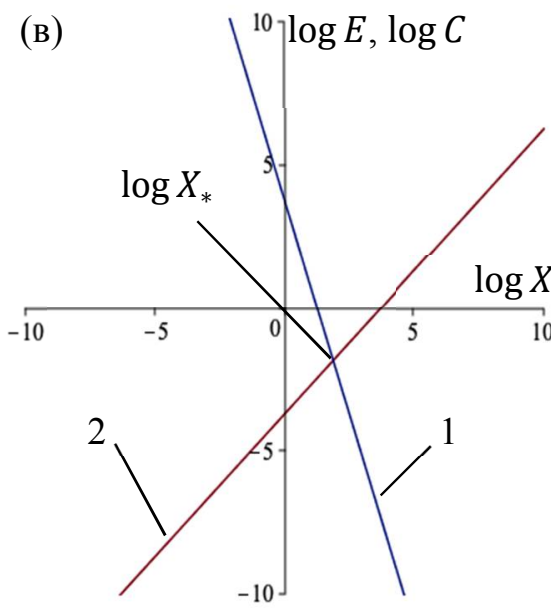

8

Рис. 3. Логарифмические графики зависимостей параметров $E$ (прямая 1) и $C$ (прямая 2) от $X$ при $n=1,2$ и $3(a-6)$ 
То есть, можно сделать следующий вывод: при малых масштабах по времени (по длине) течение неравновесное, влияние капиллярных сил мало, с другой стороны, при увеличении масштаба по времени (длине) или при больших $X$ наблюдается большое влияние капиллярных сил на характер течения, которые преобладают над неравновесностью.

В соответствии с зависимостью параметров $E$ и $C$ от $X$ при $n=2$ имеем $E=E_{0} X^{-2}$, $C=C_{0}$. Параметр $E$ при больших $X$ мал, следовательно, при $X \rightarrow \infty$ решение полной задачи для трещиновато-пористой среды несущественно отклоняется от асимптотического распределения (рис. 8, 9). При малых $X$ наблюдается неравновесный процесс - перетоки между $\Phi^{f}$ и $\Phi^{m}$ минимальны, $q^{m f} \rightarrow 0$ (рис. 4,5 ).

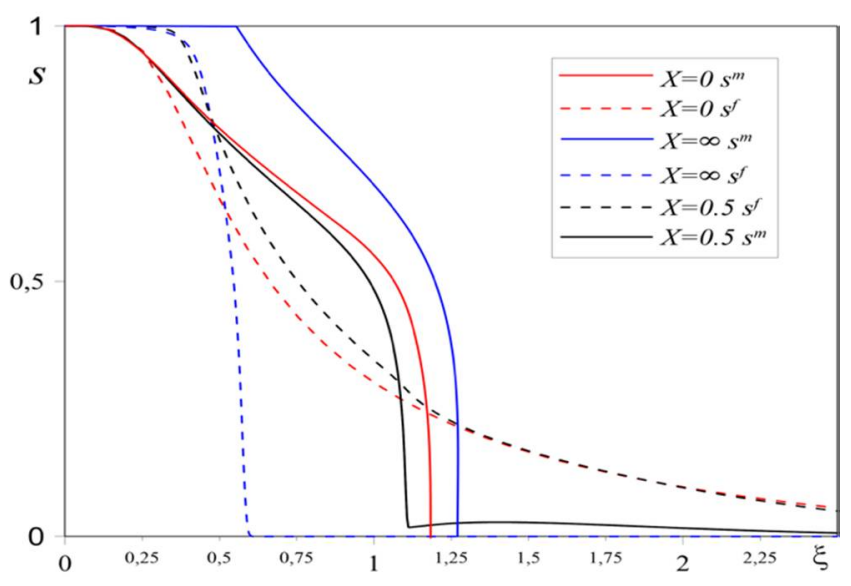

Рис. 4. Распределение насыщенности при $X=0.5$ в континуумах $\Phi^{f}$ и $\Phi^{m}$

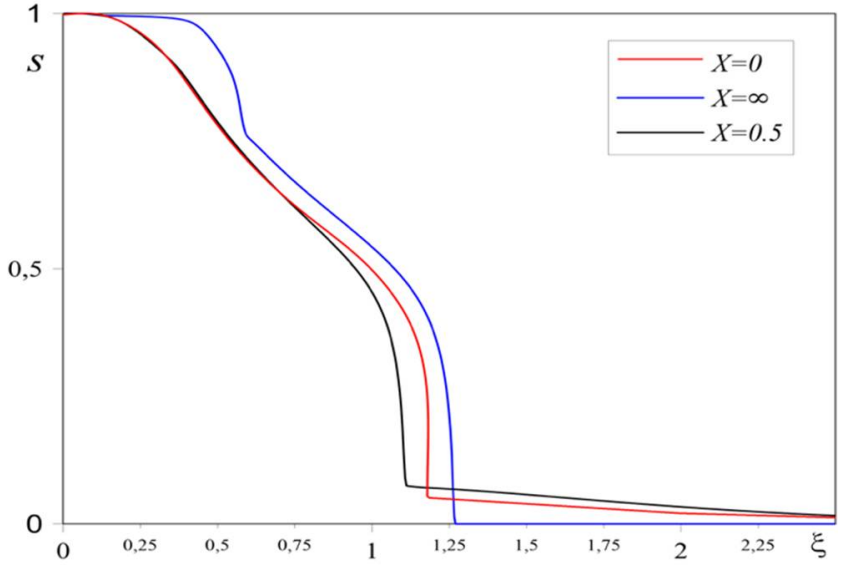

Рис. 5. Распределение эффективной насыщенности при $X=0.5$ в континуумах $\Phi^{f}$ и $\Phi^{m}$

Отметим, что протяженность фронта вытеснения (переходного слоя, в котором существенно изменяется насыщенность), образованного неравновесными процессами $(C=0$, $E \neq 0)$, с ростом $X$ убывает как $1 / X^{2}$ [4], тогда как толщина зоны вытеснения, обусловленного капиллярными силами $(C \neq 0, E=0)$, не изменяется в зависимости от $X$. Следовательно, параметры меняются непрерывно за счёт постоянного влияния капиллярного давления на распределение насыщенности в пространстве.

На рис. 4, 5 представлены распределения насыщенностей в континуумах $\Phi^{f}$ и $\Phi^{m}$ и распределение эффективной насыщенности при конечных $X$. При малых $X$, в частности, в случае $X=0.5$ (рис. 4) наблюдается неравновесность течения. Фронт вытеснения, соответствующий $\Phi^{f}$, распространяется быстрее фронта, соответствующего $\Phi^{m}$, т. к. жидкость при малых масштабах времени слабо впитывается в блоки, а распространяется в основном по трещинам.

При промежуточных значениях $X$, в частности при $X=0.5$, течение приближается к состоянию локального капиллярного равновесия. За счет действия капиллярных сил большая часть воды, попадающая в трещины, впитывается в блоки, и фронт вытеснения в блоках $\Phi^{m}$ обгоняет фронт в трещинах $\Phi^{f}$ (рис. 6,7 ). При данных $X$ жидкость начинает быстрее распространяться по блокам.

При достаточно больших $X$ между $\Phi^{f}$ и $\Phi^{m}$ устанавливается капиллярное равновесие. При этом заметно установление двух фронтов вытеснения в $\Phi^{f}$ и $\Phi^{m}$ (рис. 8). Распределение эффективной насыщенности хорошо совпадает с распределением насыщенности, соответствующим асимптотической $(X \rightarrow \infty)$ модели одинарной пористости при заданных, исходя из предположений, параметрах (рис. 9). 


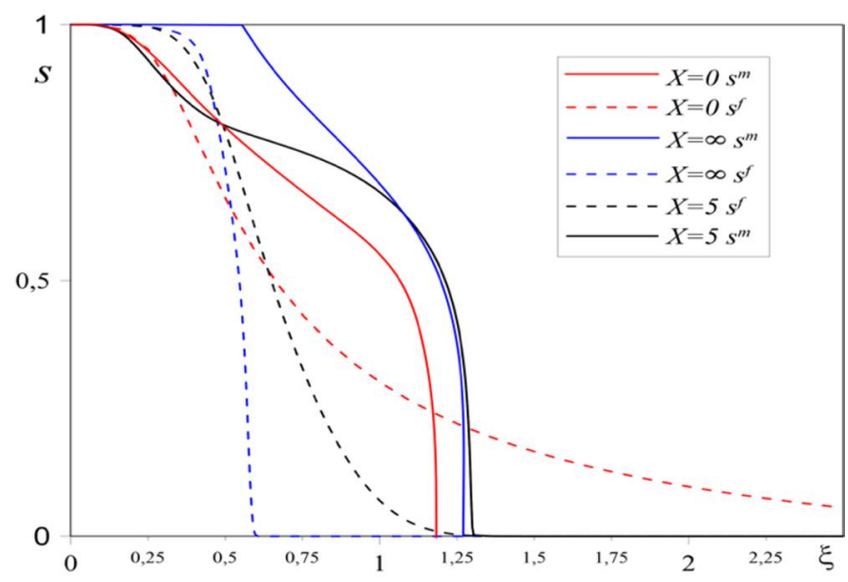

Рис. 6. Распределение насыщенности при $X=5$ в континуумах $\Phi^{f}$ и $\Phi^{m}$

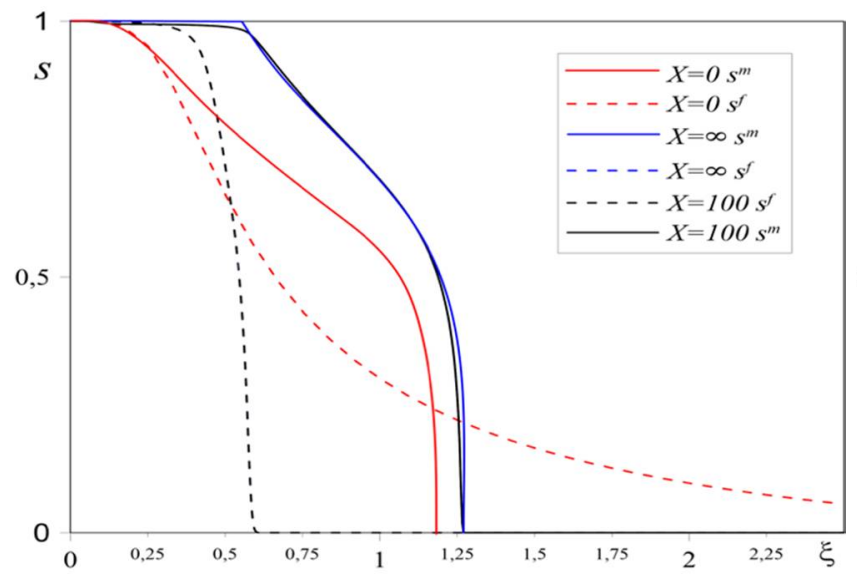

Рис. 8. Распределение насыщенности при $X=100$ в континуумах $\Phi^{f}$ и $\Phi^{m}$

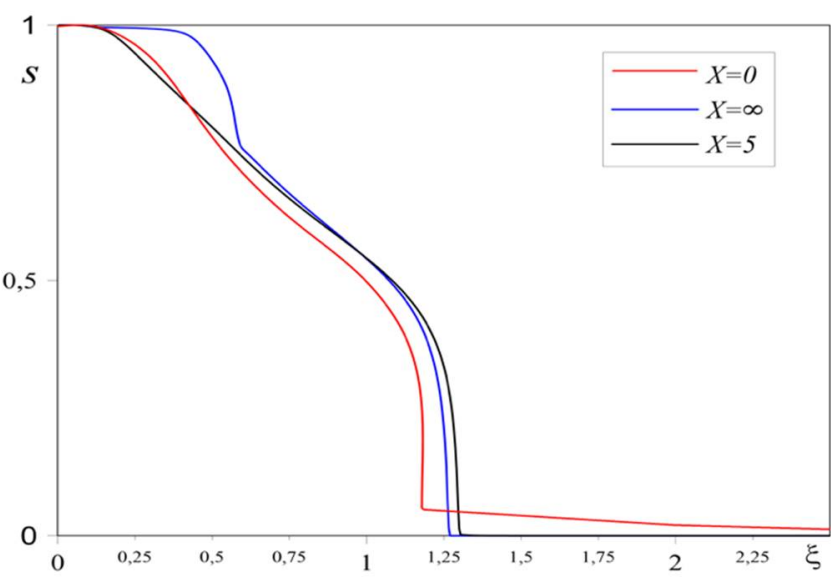

Рис. 7. Распределение эффективной насыщенности при $X=5$ в континуумах $\Phi^{f}$ и $\Phi^{m}$

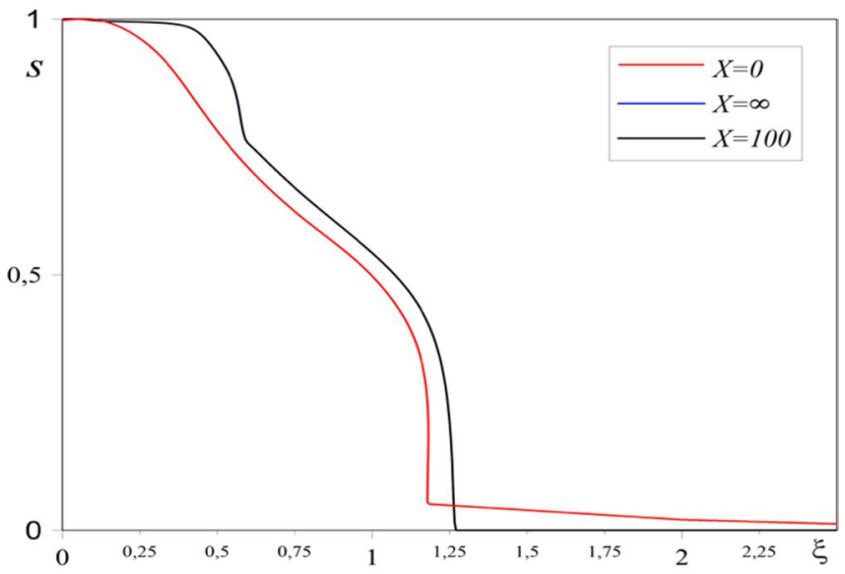

Рис. 9. Распределение эффективной насыщенности при $X=100$ в континуумах $\Phi^{f}$ и $\Phi^{m}$

\section{6. Заключение}

Исследованы решения обобщенной задачи Баклея - Леверетта, описывающей двухфазное вытеснение в трещиновато-пористой среде. Определены критерии подобия, характеризующие влияние капиллярного давления на распределение параметров течения в пространстве и на локальное равновесие между двумя континуумами - трещинами и блоками. Показано, что зависимость параметров от характерного пространственного масштаба течения (или его характерного времени) определяется симметрией течения, т.е. параметром $n$. В частности, при $n=2$ один из параметров, $C$, характеризующий влияние капиллярных сил на распределение в пространстве, остаётся неизменным, а параметр $E$, характеризующий неравновесность течения, квадратично убывает с увеличением характерного масштаба пространства и времени. Показано, что на начальных этапах закачки жидкости в трещиноватопористую среду задача распадается на две независимые задачи Баклея-Леверетта для каждого из континуумов. Получены уравнения эффективной модели одинарной пористости, описывающие параметры равновесного течения на поздних этапах закачки, т. е. в предельном случае бесконечно большого пространственного масштаба. Проведены расчеты переходных режимов течения на промежуточных этапах закачки жидкости. Обнаружено, что в условиях локального равновесия течение в трещиновато-пористой среде может сопровождаться фор- 
мированием последовательности двух фронтов вытеснения. Продемонстрировано, что распределения параметров течения в переходных слоях, соответствующих данным фронтам, определяется не только капиллярным давлением, но и неравновесными перетоками между трещинами и блоками.

\section{Благодарности и ссылки на гранты}

Работа выполнена при финансовой поддержке Совета по грантам Президента РФ (МД3567.20181).

\section{Литература}

1. Баренблатт Г.И., Желтов Ю.П., Кочина И.Н. Об основных представлениях теории фильтрации однородных жидкостей в трещиноватых породах // ПММ. 1960. Т. 24. Вып. 5. С. 852-864.

2. Buckley S.E., Leverett M.C. Mechanism of fluid displacement in sands // Trans. AIME. 1942. V. 146. Pp. 107-116.

3. Баренблат Г.И., Ентов В.М., Рыжик В.М. Движение жидкостей и газов в природных пластах. М.: Недра, 1984. 211 с.

4. Афанасьев А.А. Осредненная асимптотическая модель двухфазной фильтрации в трещиноватопористых средах // Изв. РАН МЖГ. 2019. № 5. С. 83-92

5. Kazemi, H., Merill, L.S., Porterfield, K.L., Zeman, P.R., 1976. Numerical simulation of water-oil flow in naturally fractured reservoirs. SPE J. 16(6), pp. 317-326.

6. Brooks A.N., Corey A.T. Hydraulic properties of porous media. Hydrology Papers 3. Colorado State University, 1964.

7. Afanasyev A. Reservoir simulation with MUFITS code: Extension for double porosity reservoirs and flows in horizontal wells // Energy Proc. 2017. V. 125. Pp. 596-603.

Статья поступила в редакцию 17 октября 2019 г. 